\title{
AVALIAÇÃO DA INTEGRIDADE ANATÔMICA POR EXAME DE ULTRASSOM E FUNCIONAL PELO ÍNDICE DE CONSTANT \& MURLEY DO MANGUITO ROTADOR APÓS REPARO ARTROSCÓPICO
}

\author{
EVALUATION OF ANATOMICAL INTEGRITY USING ULTRASOUND IMAGES, \\ AND FUNCTIONAL INTEGRITY BY THE CONSTANT \& MURLEY SCORE, \\ OF THE ROTATOR CUFF FOLLOWING ARTHROSCOPIC REPAIR
}

Glaydson Gomes Godinho', Flavio de Oliveira França ${ }^{2}$, José Marcio Alves Freitas ${ }^{3}$, Fábio Nagato Watanabe ${ }^{4}$, Leonardo Oliveira Nobre ${ }^{4}$, Manoel Augusto de Almeida Neto ${ }^{4}$, Marcos André Mendes da Silva ${ }^{4}$

\section{RESUMO}

Objetivo: Avaliar os resultados funcionais e anatômicos do tratamento cirúrgico via artroscópica nas roturas completas do manguito rotador usando imagens de ultrassom e o índice funcional de Constant e Murley, investigando a correlação entre eles. Métodos: Avaliados 100 pacientes (110 ombros). Seguimento médio de 48,8 \pm 33,28 (12 a 141) meses. Média de idade de $60,25 \pm 10,09$ (36 a 81) anos. Rotura isolada do tendão supraespinal esteve presente em 85 casos (77\%); em associação com o infraespinal, em 20 (18\%), e associado ao subescapular, em quatro ombros (4\%). A associação de lesões supra-espinal, infraespinal e subescapular foi encontrada em um ombro (1\%). De acordo com DeOrio e Cofield, as lesões foram classificadas em pequenas/médias em 85 ombros (77\%) e, grandes/extensas em 25 (23\%). Avaliação clínica de resultados realizada de acordo com critérios de Constant e Murley. Resultados do ultrassom (US) se referem aos laudos emitidos por diferentes radiologistas. Análise estatística de acordo com os métodos Qui-quadrado, teste exato de Fisher, teste $t$ de Student, correlação de Pearson, Kruscall-Wallis e regressão logística (significância $\mathrm{p}<0,05$ ). Resultados: Média da avaliação de Constant de 85,3 \pm 10,06 nos ombros normais e 83,96 $\pm 8,67$ nos operados $(\mathrm{p}=0,224)$. Excelentes e bons resultados observados em 74 ombros (67\%), satisfatórios e regulares em 32 (29\%) e maus em quatro (4\%). Avaliação por ultrassonografia evidenciou 38 ombros com rerotura (35\%) e integridade em 71 (65\%). Nos 74 (67\%) ombros com excelentes/bons resultados, 22 (30\%) apresentaram laudo ultrassonográfico de re-rotura $(\mathrm{p}=0,294)$. Nos quatro (4\%) ombros com maus resultados, dois (50\%) apresentaram laudos

\section{ABSTRACT}

Objective: To evaluate the functional and anatomic results of surgical treatment under arthroscopy in cases of rupture of the rotator cuff using ultrasound images and the Constant and Murley functional score to investigate the correlation between them. Methods: 110 patients (110 shoulders) were evaluated. The medium follow up was $48.8 \pm 33.28$ months (12 to 141 months). The average age was $60.25 \pm 10.09$ (36 to 81 years). Isolated rupture of the supraspinal tendon occurred in 85 cases (77\%), associated with infraspinatus in 20 cases (18\%) and subscapularis in four shoulders (4\%). Associated tear lesions of the supraspinatus, infraspinatus and subscapularis was found in one shoulder (1\%). The lesions were classified according to the DeOrio and Cofield score as small/medium in 85 shoulders (77\%) and large/massive in 25 (23\%). The clinical results were assessed according to the Constant and Murley criteria. The results of the ultrasound (US) refer to the reports of several radiologists. Statistical analysis was carried out according to the chi-square methods, Fisher's exact test, Student T test, Pearson, Kruscall-Wallis correlation and logistic regression (significance value $p$ <0.05). Results: the Constant evaluation average was $85.3 \pm 10.06$ (46 to 99) in the normal shoulders and $83.96 \pm$ 8.67 (59 to 99) in the operated shoulders $(p=0.224)$. Excellent and good results were found in 74 shoulders (67\%), satisfactory and adequate results in 32 (29\%) and poor results in 4 (4\%). The ultrasound evaluation showed 38 shoulders with re-rupture (35\%), absence of rupture in 71 (65\%). Of the 74 (67\%) shoulders with excellent/good results, 22 (30\%) presented re-rupture in the ultrasound report ( $p=0.294)$. Of the four shoulders

1 - Chefe do Grupo do Ombro do Hospital Ortopédico e Hospital Belo Horizonte, Mestre e Doutor em Ortopedia.

2 - Cirurgião Assistente do Grupo de Ombro do Hospital Ortopédico e Hospital da Polícia Militar de Minas Gerais.

3 - Cirurgião Assistente do Grupo de Ombro do Hospital Ortopédico e Hospital Belo Horizonte.

4 - Residentes do Grupo de Ombro do Hospital Ortopédico e Hospital Belo Horizonte. 
de tendões íntegros ( $\mathrm{p}=0,294)$. Conclusão: Não há correlação estatisticamente válida entre o diagnóstico ultrassonográfico e o método clínico de avaliação de resultados de pacientes submetidos ao reparo artroscópico de roturas completas do manguito rotador. Os resultados clínicos nos reparos das roturas completas do manguito rotador por via artroscópica apresentam alto nível de recuperação funcional (Constant 83,96) quando comparado com o ombro contralateral. Os laudos de ultrassom pós-operatórios apresentam alta porcentagem de re-rotura (35\%). A força pós-operatória é maior nos pacientes com menos de 60 anos de idade $(\mathrm{p}=0,002)$ e em casos de lesões menores ou iguais a $3 \mathrm{~cm}(\mathrm{p}=0,003)$.

Descritores - Bainha rotadora; Artroscopia; Ultrassonografia

\section{INTRODUÇÃO}

As roturas do manguito rotador (MR) são frequentes na população acima dos 50 anos de idade, sendo uma doença de etiologia multifatorial, com característica de lesão degenerativa na maioria dos casos, incidindo em 10 a $90 \%$ da população $0^{(1,2)}$. O diagnóstico está fundamentado na avaliação clínica e por métodos de imagem. Fatores como idade fisiológica e demanda funcional determinarão se o tratamento será conservador ou cirúrgico (reparo aberto ou artroscópico). A avaliação funcional e estrutural pós-operatória tem sido objeto de discussão ${ }^{(3,4)}$, sendo a maioria dos estudos com seguimento pequeno e com baixa casuística. Muitas dificuldades são encontradas na avaliação pós-operatória do manguito rotador por ultrassonografia, dentre elas, a compreensão da variação anatômica. Embora esse exame de imagem tenha elevados índices de sensibilidade e especificidade préoperatória, o mesmo não é evidenciado em avaliações pós-cirúrgicas. Apesar de impreciso ${ }^{(5-7)}$, é empregado frequentemente como exame de imagem para o diagnóstico de re-roturas do manguito rotador, por ser um exame de baixo custo, não invasivo, de fácil acesso à população, e, ainda, permitir a avaliação do ombro contralateral ${ }^{(8)}$. Esse método pode identificar os tendões envolvidos e a extensão da lesão ${ }^{(6)}$, porém, a qualidade da imagem é fundamental para o diagnóstico ${ }^{(9)}$.

O reparo artroscópico das lesões do manguito rotador apresenta elevados índices de bons e excelentes resultados clínicos ${ }^{(1,4,5,10,11)}$, ainda que, em muitos casos, não ocorra uma cicatrização adequada do tendão $0^{(3)}$. A re-rotura é uma das complicações encontradas. Estudos utilizando escores funcionais e exames de imagem demonstram correlações incoerentes entre função e laudos de re-rotura. Em razão disso, não existe um padrão bem
(4\%) with poor results, two (50\%) presented intact tendons ( $p$ $=0.294)$. Conclusion: There is no statistically valid correlation between the ultrasound diagnosis and the clinical evaluation of results in patients who underwent arthroscopic repair to treat full tear lesions of the rotator cuff. The clinical results in complete rotator cuff repairs under arthroscopy present a high level of functional recovery (Constant 83.96) when compared with the contralateral shoulder. The postoperative ultrasound image reports present a high percentage of re-rupture (35\%); The postoperative strength is higher in patients aged under 60 years $(p=0.002)$ and in cases of lesions less than or equal to $3 \mathrm{~cm} .(p=0.003)$.

Keywords - Rotator cuff; Arthroscopy; Ultrasonography

definido que correlacione capacidade funcional e integridade anatômica dos tendões ${ }^{(3)}$. As imagens de re-rotura ainda são um desafio para o radiologista. Há necessidade de se padronizar o que é uma imagem de re-rotura, porque as definições desta lesão e de sua extensão variam significativamente na literatura ${ }^{(3)}$. Contudo, o alto custo da ressonância nuclear magnética (RNM), o grau de dificuldade para compreender a anatomia pós-cirúrgica do manguito rotador através deste exame e a inviabilidade de realizar artroscopia (padrão ouro) em todos os pacientes em acompanhamento pós-operatório, tornam o US uma opção viável. Por ser um exame de imagem examinador dependente, o US deveria ser realizado por um mesmo radiologista. Infelizmente, esta não é a realidade do nosso país, e muitas vezes por motivos que fogem às demandas do médico, o exame é feito de forma aleatória.

O objetivo deste estudo foi avaliar a integridade anatômica e funcional após reparo artroscópico das lesões completas do manguito rotador, utilizando imagens de ultrassom e escore funcional de Constant e Murley ${ }^{(12)}$, investigando o índice de concordância entre eles.

\section{CASUÍSTICA E MÉTODOS}

Foi realizado um estudo prospectivo com avaliação funcional e de US. Foram realizadas 1.531 cirurgias no período de setembro de 1996 a maio de 2007, nos Hospitais Ortopédico e Belo Horizonte, em Belo Horizonte, Minas Gerais.

Foram incluídos no estudo pacientes em pós-operatório de reparo artroscópico de lesão completa do manguito rotador, com seguimento mínimo de um ano. Todos os pacientes foram submetidos ao escore funcional de Constant e Murley e ultrassom (US) pós-cirúrgico (Figuras $1 \mathrm{~A}$ e $1 \mathrm{~B})$. 


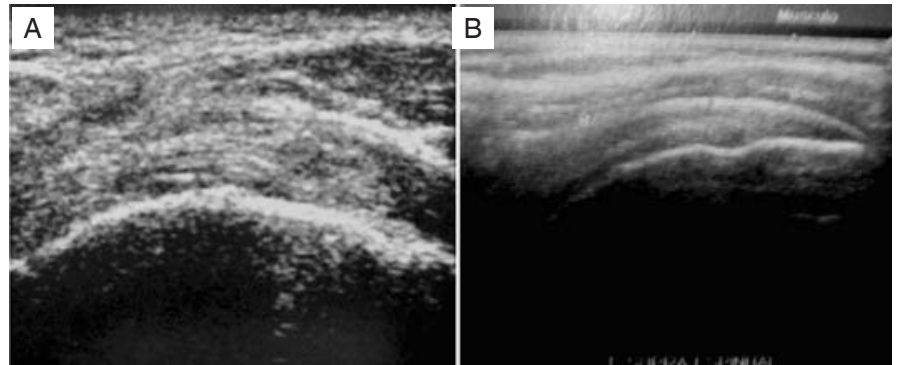

Figura 1 - A) Imagem de ultrassom demonstrando alteração anatômica decorrente de área de cicatriz no tendão supraespinal. B) Imagem de ultrassom evidenciando integridade anatômica

Os critérios de exclusão foram o tempo de seguimento menor que um ano, artrose glenoumeral com degeneração articular avançada, tendinite calcária, artrite reumatoide, capsulite adesiva, sequelas de fraturas, pacientes não localizados ou com documentação insuficiente.

Selecionados 147 pacientes (159 ombros), dos quais foram excluídos 47 pacientes (49 ombros) por falta de exame de US pós-operatório, avaliação funcional incompleta ou ambos. Restaram 100 pacientes (110 ombros), 36 (33\%) do sexo masculino e 74 (67\%) femininos. Seguimento médio de 48,8 \pm 33,28 meses (variando de 12 a 141 meses). A média de idade do grupo estudado foi 60,25 \pm 10,09 anos (variando de 36 a 81) (Figura 2). O lado direito foi acometido em 86 ombros (78\%), e o esquerdo em 24 ombros (22\%). Dez pacientes (10\%) tinham lesões bilaterais. O lado dominante correspondeu a 88 ombros (80\%), e não dominantes 22 ombros (20\%). Trinta e quatro por cento dos pacientes eram praticantes de esportes e 66 (66\%), não.

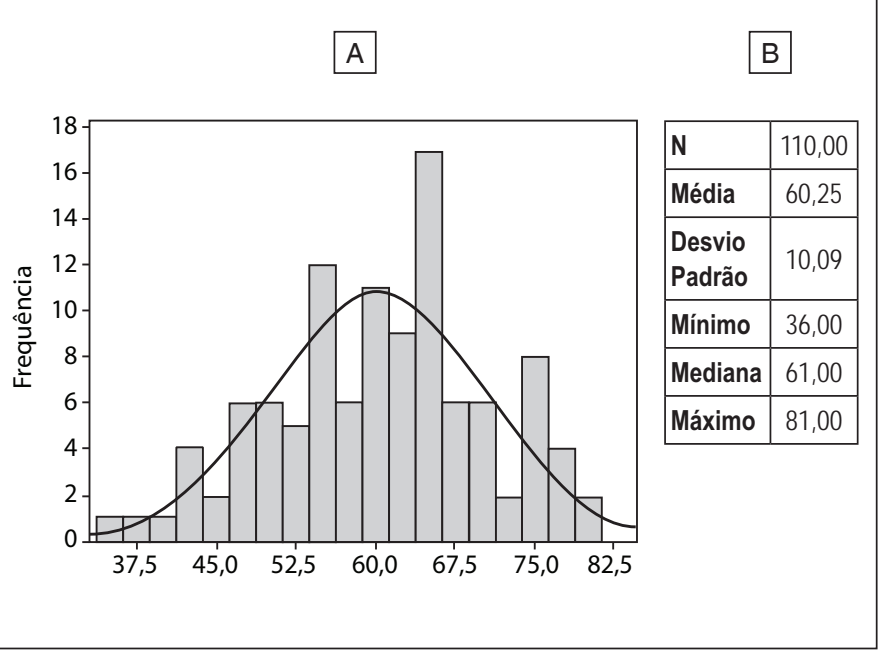

Figura 2 - A) Distribuição populacional conforme a idade. B) Caracterização populacional conforme a idade
No pré-operatório, foi constatado que na topografia dos tendões envolvidos, o supra-espinal esteve presente em 110 ombros (100\% dos casos). A rotura isolada do tendão supra-espinal esteve presente em 85 ombros (77\%), em associação com o infraespinal (lesão posterossuperior) em 20 ombros (18\%), e associado ao subescapular (lesão anterossuperior) em quatro ombros (4\%). A associação de lesão do tendão supra-espinal com o infraespinal e o subescapular foi encontrada em um ombro (1\%) (Figura 3).

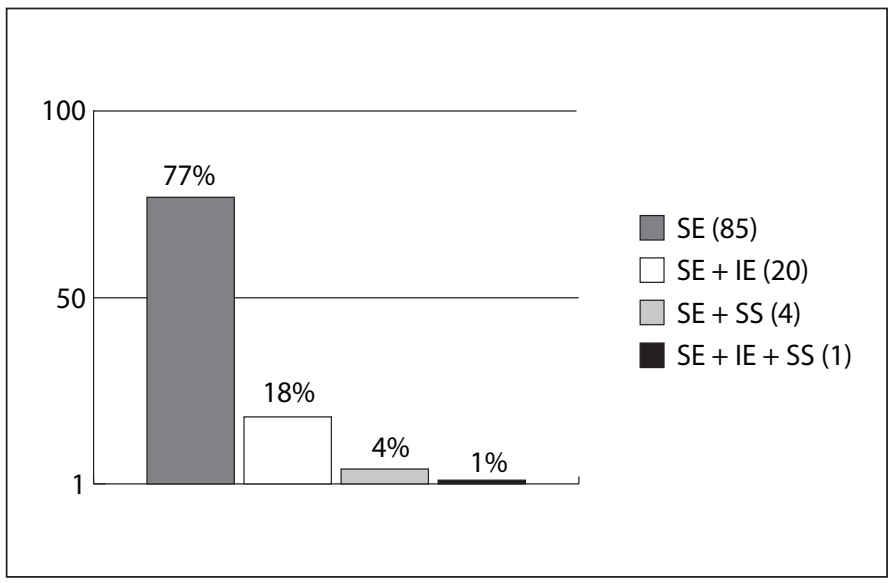

Figura 3 - Tendões acometidos

A extensão das lesões no sentido anteroposterior, medida durante o procedimento artroscópico, após seu desbridamento, variou de $0,5 \mathrm{~cm}$ a $5,4 \mathrm{~cm}$. De acordo com DeOrio e Cofield ${ }^{(13)}$, foram classificadas em pequenas $(\leq 1 \mathrm{~cm})$, presentes em $14(13 \%)$ ombros, médias $(1-3 \mathrm{~cm})$ em $71(65 \%)$, grandes $(3-5 \mathrm{~cm})$ em 23 (21\%) e extensas $(>5 \mathrm{~cm})$ presentes em dois $(2 \%)$ ombros (Figura 4).

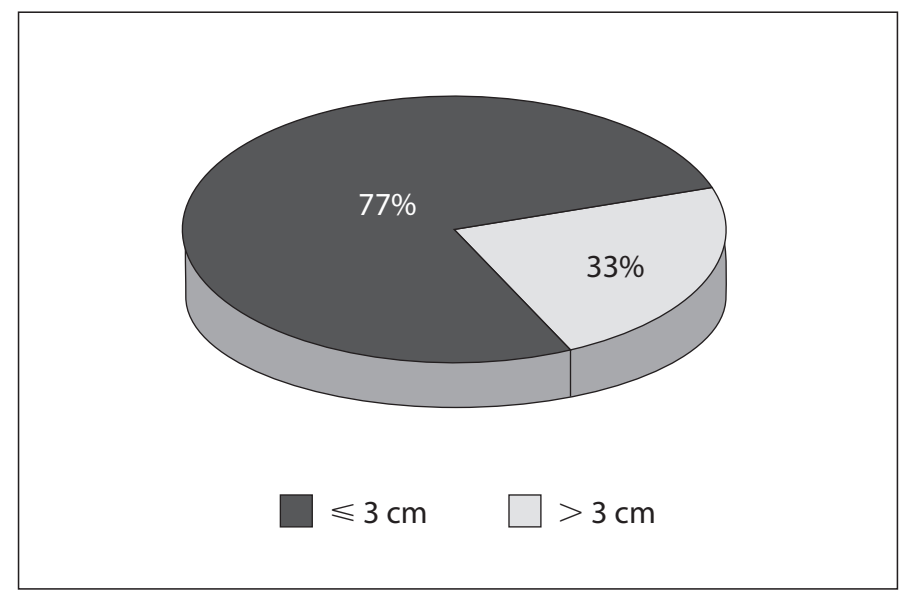

Figura 4 - Extensão da lesão no sentido anteroposterior após o desbridamento tendíneo 
A avaliação do tendão da cabeça longa do bíceps no intraoperatório demonstrou 63 (57\%) tendões normais e cinco (5\%) rotos. Os demais apresentavam algum grau de degeneração, e foi realizada tenólise em dois (2\%), tenotomia em 31 (28\%) e tenodese em nove (8\%).

Foi realizada descompressão subacromial (acromioplastia anteroinferior) em 108 (98\%) ombros.

A avaliação clínica de resultados foi realizada de acordo com os critérios de Constant e Murley ${ }^{(12)}$, que utilizam dois parâmetros subjetivos (dor e atividades de vida diária) e dois objetivos (amplitude de movimento e força) totalizando 100 pontos (ombro assintomático, com função completa e força de aproximadamente $11 \mathrm{~kg})$. A dor pode estar ausente (15 pontos), leve (10 pontos), moderada (5 pontos) e intensa (0). As atividades da vida diária somam 20 pontos. Trabalho integral sem limitação (4 pontos), atividade recreativa sem limitação (4 pontos), sem perturbação do sono pela dor (2 pontos) e posicionamento da mão no espaço (10 pontos quando atinge o nível acima da cabeça). A função é avaliada segundo a amplitude de movimento (ADM), sendo 10 pontos para cada avaliação integral (rotação medial, rotação lateral, abdução e flexão), somando 40 pontos. A força é fornecida em "pounds” (força em kg multiplicada por uma constante de 2,2). Assim, o padrão normal se refere a um indivíduo de 25 anos, assintomático, com força de 25 "pounds" (aproximadamente $11 \mathrm{~kg})$. Para tal, foi utilizada uma balança doméstica de ponto fixo (Figuras 5A e 5B), com marcação limitada a $12 \mathrm{~kg}$, registrando o valor mensurado através do teste de Jobe ${ }^{(12,14)}$. Segundo Boehm ${ }^{(15)}$, os resultados encontrados com o método de Constant podem ser: excelentes (91-100), bons (81-90), satisfatórios (71-80), regulares $(61-70)$ ou maus $(<60)$.

Os resultados da ultrassonografia se referem a laudos emitidos por diferentes radiologistas e equipamentos com transdutores de potências variadas (7,5 a 15MHz).

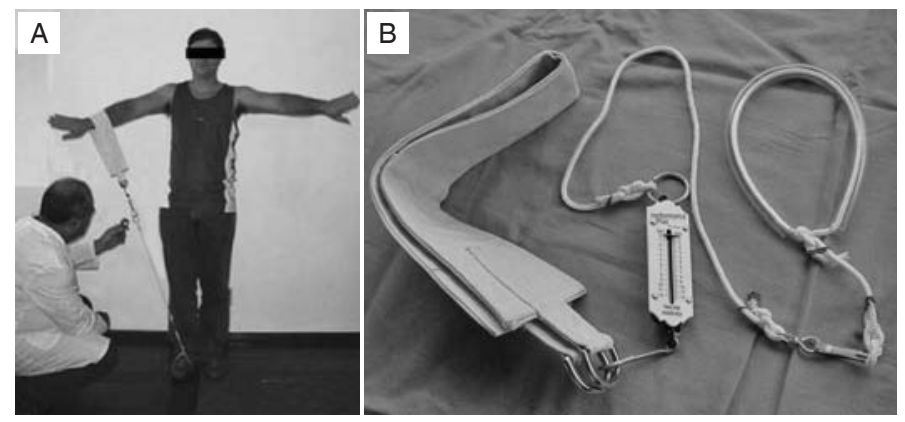

Figura 5 - A) Posicionamento para a medida da força segundo Constant e Fisher. B) Dispositivo com dinamômetro convencional de ponto fixo limitado a $12 \mathrm{~kg}$
Os exames foram realizados no pós-operatório, com intervalos entre um e dois anos em 52 ombros (47\%); entre dois e cinco anos em 31 ombros (28\%); mais de cinco anos, 27 ombros (25\%).

A análise estatística foi feita de acordo com os métodos Qui-quadrado, teste exato de Fisher, teste $t$ de Student, correlação de Pearson, Kruscall-Wallis e regressão logística (valor de significância p <0,05).

\section{RESULTADOS}

A média da avaliação de Constant encontrada foi de $85,3 \pm 10,06$ (46 a 99) nos ombros normais e 83,96 \pm $8,67$ (59 a 99) nos ombros operados ( $p=0,224)$. Excelentes e bons resultados foram observados em 74 ombros (67\%), satisfatórios e regulares em 32 (29\%) e maus resultados em quatro (4\%).

Excluindo os casos bilaterais, a média da força pósoperatória ipsilateral foi $5,53 \mathrm{~kg} \pm 2,34$ (2 a 11) e a contralateral foi $5,88 \mathrm{~kg} \pm 2,45(0,6$ a 11$)(p=0,091)$. A força pós-operatória média foi $6,38 \mathrm{~kg} \pm 2,53$ nos pacientes com faixa etária entre 41-60 anos, e 4,78kg \pm 2,23 nos pacientes acima dos 60 anos $(\mathrm{p}=0,002)$. Nas lesões menores ou iguais a 3cm, 85 ombros (77\%) apresentaram força pós-operatória média de 5,64kg \pm 2,41. Nas maiores que $3 \mathrm{~cm}, 25$ ombros (23\%) demonstraram $4,00 \mathrm{~kg} \pm 2,03(\mathrm{p}=0,003)$.

Não houve diferença estatisticamente significativa da média das forças pós-operatórias entre os pacientes que apresentaram laudos ultrassonográficos de re-rotura e os que apresentaram integridade anatômica $(p=0,256)$ (Figura 6).

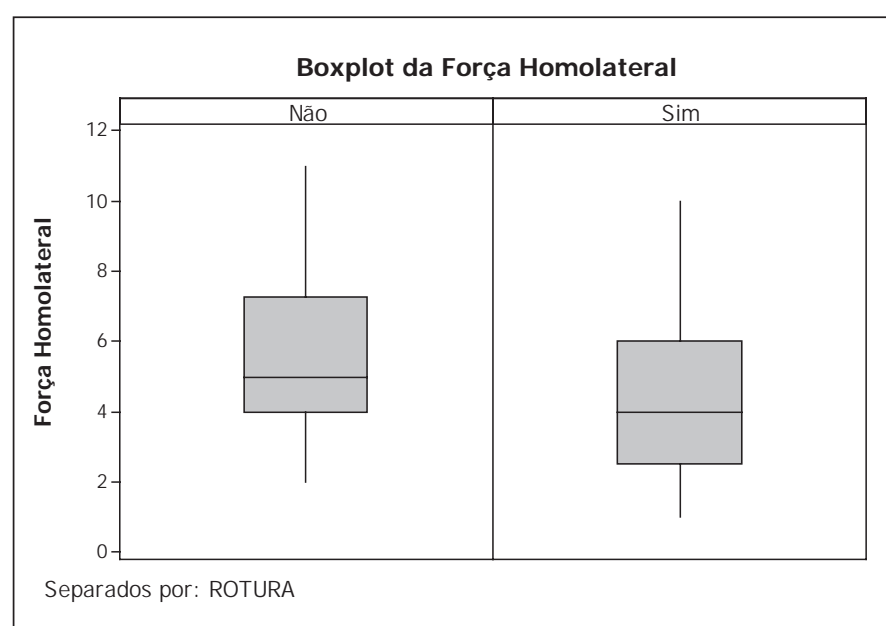

Figura 6 - Estatística descritiva evidenciando a diferença sem significância, entre as médias das forças pós-operatórias com e sem re-rotura tendínea ao US. $(p=0,256)$. Não: ausência de re-rotura; Sim: presença de re-rotura 
A média do escore de Constant nos pacientes em que foi realizada a tenodese da cabeça longa do bíceps, foi $86,73 \pm 9,42$ e, 78,10 $\pm 10,60$ nos casos com tenotomia $(\mathrm{p}=0,035)$.

Em 81 pacientes (74\%) avaliados não houve nenhuma queixa de dor enquanto que 29 (26\%) apresentaram algum grau de dor residual. Destes, 20 (17\%) queixavam dor leve, cinco (5\%) dor moderada e quatro (4\%) dor severa. Nos pacientes com excelentes e bons resultados, oito (11\%), apresentaram queixa de dor.

A avaliação por ultrassonografia evidenciou 38 ombros (35\%) com re-rotura, ausência de rotura em 71 ombros (65\%) e um diagnóstico inconclusivo que foi excluído.

A ocorrência simultânea dos resultados segundo a classificação de Constant e os laudos ultrassonográfico estão representados no Quadro 1 ( $\mathrm{P}=0,294)$ (Quadro 1).

Quadro 1 - Correlação entre a avaliação funcional de Constant (categorizado por Bohem) ${ }^{(15)}$ e os resultados da ultrassonografia

\begin{tabular}{|c|c|c|c|}
\hline \multirow{2}{*}{ Constant } & \multicolumn{2}{|c|}{ Ultrassonografia } & \multirow{2}{*}{ Total } \\
\cline { 2 - 3 } & Re-rotura & Íntegros & \\
\hline $\begin{array}{c}\text { Excelente } \\
\text { Bom }\end{array}$ & 22 & 51 & 73 \\
\hline $\begin{array}{c}\text { Satisfatório } \\
\text { Regular }\end{array}$ & 14 & 18 & 32 \\
\hline Mau & 2 & 2 & 4 \\
\hline Total & 38 & 71 & 109 \\
\hline
\end{tabular}

A correlação entre os achados do ultrassom e o tempo de seguimento está representada no Quadro 2 $(\mathrm{P}=0,133)$.

A ocorrência de re-roturas (ultrassonografia) de acordo com a extensão das lesões está representada na Quadro $3(\mathrm{P}=0,531)$.

Quadro 2 - Correlação entre o tempo de seguimento e os resultados da ultrassonografia

\begin{tabular}{|c|c|c|c|}
\hline \multirow{2}{*}{ Seguimento } & \multicolumn{2}{|c|}{ Ultrassonografia } & \multirow{2}{*}{ Total } \\
\cline { 2 - 3 } & Re-rotura & Íntegros & \\
\hline 1 a 2 anos & $14(27 \%)$ & $38(73 \%)$ & $52(100 \%)$ \\
\hline 2 a 5 anos & $11(35 \%)$ & $20(65 \%)$ & $31(100 \%)$ \\
\hline$>5$ anos & $13(50 \%)$ & $13(50 \%)$ & $26(100 \%)$ \\
\hline
\end{tabular}

Quadro 3 - Correlação entre a extensão da lesão do manguito rotador e os resultados da ultrassonografia

\begin{tabular}{|c|c|c|c|}
\hline \multirow{2}{*}{ Extensão da lesão } & \multicolumn{2}{|c|}{ Ultrassonografia } & \multirow{2}{*}{ Total } \\
\cline { 2 - 3 } & Re-rotura & Íntegros & \\
\hline$\leq 3 \mathrm{~cm}$ & $30(35 \%)$ & $55(65 \%)$ & $85(100 \%)$ \\
\hline$>3 \mathrm{~cm}$ & $8(33 \%)$ & $16(67 \%)$ & $24(100 \%)$ \\
\hline
\end{tabular}

\section{DISCUSSÃO}

A ultrassonografia é utilizada amplamente para diagnóstico das lesões do manguito rotado ${ }^{(9,16)}$. Entretanto, seus resultados ficam comprometidos quando se analisa um ombro operado ${ }^{(16,17)}$. Alguns autores afirmam que este método diagnóstico pode apresentar altos índices de sensibilidade, especificidade e precisão, mesmo nestas condições, quando feitos por profissionais experientes $^{(5-7,18,19)}$. Através deste estudo, não evidenciamos correlação coerente entre a análise estrutural dos tendões (US) e o resultado funcional (Constant). Observamos valores de até $20 \%$ de re-rotura em pacientes com excelentes e bons resultados funcionais, o que é também citado na literatura ${ }^{(1,3,4,17)}$. A avaliação funcional e estrutural pós-operatória tem sido objeto de discussão ${ }^{(3,4,17)}$. Observa-se que os estudos de reparos por via artroscópica apresentam resultados de seguimentos e casuísticas pequenas $^{(3,6,7,20)}$. A inadequada compreensão e a falta de padronização dos achados ultrassonográficos póscirúrgicos $^{(21)}$ podem justificar, mesmo que parcialmente, nossos achados incoerentes. Crass et $a^{(21)}$ descreveram a distorção anatômica decorrente das aderências entre o músculo deltoide e o manguito rotador, a presença de tecido de granulação e a mudança no sentido das fibras como obstáculos verdadeiros para o diagnóstico preciso (aquilo que realmente representa re-rotura), sugerindo a necessidade de um método mais eficaz de avaliação por imagens. Características semelhantes foram citadas em outros trabalhos ${ }^{(16,20)}$. Furtschegger e Resch ${ }^{(16)}$ diante de tais limitações, delinearam um estudo na tentativa de definir critérios ultrassonográficos pós-cirúrgicos para as lesões do manguito rotador. Eles enfatizaram a não visualização do tendão e a sua descontinuidade ecogênica como fatores determinantes para o diagnóstico de lesão.

A padronização do equipamento e da técnica ultrassonográfica é recomendado por vários autores ${ }^{(3,5,6)}$. Contudo, tal prática acadêmica não reflete a realidade 
do exercício clínico. Nem sempre, paciente e médico podem confiar seus exames a apenas poucos profissionais muito experientes ou, a centros de excelência, com equipamentos de última geração. Assim, os estudos realizados em condições especiais supervalorizam o método, de forma genérica e distante da realidade. O US diagnóstico tem sofrido avanços significativos a serem reconsiderados.

Adotamos o protocolo funcional de Constant e Murley ${ }^{(12)}$ e agrupamos nossos resultados conforme sugerido por Boehm ${ }^{(15)}$, ao contrário de alguns autores que utilizam protocolos que enfatizam critérios mais subjetivos $^{(3,8,17,22)}$. Na soma deste protocolo, 25 pontos são atribuídos à força, o que corresponde a uma correlação direta com a integridade anatômica do tendão ${ }^{(4,23)}$. Na presente série, encontramos uma média absoluta do escore de Constant e Murley ${ }^{(12)}$ nos ombros operados de 83,96 $\pm 8,67$. Gartsman et al ${ }^{(23)}$ encontraram valor semelhante $(83,6)$ em seu estudo, avaliando 73 pacientes com um seguimento de dois anos. Eles também compararam a força média pré-operatória com a força média pós-operatória, encontrando um aumento médio de 2,9kg, com significância estatística.

No presente estudo, comparamos a média da força pós-operatória ipsilateral $(5,53 \mathrm{~kg} \pm 2,34)$ com a contralateral $(5,88 \mathrm{~kg} \pm 2,45)$ e não encontramos significância estatística ( $\mathrm{p}=0,091)$, demonstrando equivalência entre as mesmas. Também não foi observada diferença significativa entre a média da força pós-operatória nos pacientes que apresentavam laudos ultrassonográficos de re-rotura $(4,77 \mathrm{~kg} \pm 2,45)$, com aqueles que apresentavam laudos de tendões íntegros $(5,50 \mathrm{~kg} \pm 2,39)$ $(p=0,126)$.

Avaliamos a correlação entre a extensão das lesões e a força pós-operatória, encontrando melhores resultados nas lesões menores ou iguais a $3 \mathrm{~cm}$. A média de força observada nestes pacientes foi de $5,64 \mathrm{~kg} \pm 2,03$, contra $4,0 \mathrm{~kg} \pm 2,41$ para aqueles com lesões maiores que $3 \mathrm{~cm}$ $(\mathrm{p}=0,003)$.

Em 39 pacientes (41 ombros) documentamos a força pré-operatória com o objetivo da realização de um estudo prospectivo. Neste grupo, a variação entre as médias das forças pré e pós-cirúrgicas, foi de 3,82kg para 5,05kg ( $\mathrm{p}<0,0001)$, respectivamente.

Mack et $a l^{(20)}$ encontraram dor como sintoma residual em $26 \%$ dos pacientes com excelentes e bons resultados submetidos à cirurgia via aberta. Observamos a presença de dor em graus variáveis, em $26 \%$ dos casos por nós estudados. Entre os 74 ombros (67\%) com excelentes e bons resultados funcionais, oito ombros (11\%) apresentavam dor, porém, de leve intensidade.

A artroscopia para as lesões do manguito rotador apresenta elevados índices de bons e excelentes resultados ${ }^{(1,4,5,10,11)}$. A re-rotura é uma das complicações relatadas em uma frequência de $20-65 \%$ dos casos $^{(1,4,17)}$. Através de uma avaliação com ultrassonografia com dois anos de pós-operatório, utilizando reparo aberto do manguito rotador em lesões grandes e extensas, Galatz et $a l^{(17)}$ encontraram $94 \%$ de novas lesões. DeFranco et $a l^{(3)}$ encontraram $40 \%$ de re-roturas em três anos de seguimento pós-reparo artroscópico de lesões menores que $3 \mathrm{~cm}$, utilizando o US. Harryman et $\mathrm{al}^{(8)}$ afirmaram que o US foi capaz de diagnosticar $65 \%$ dos tendões com integridade, após cinco anos da cirurgia. Sugaya et $a l^{(22)}$ encontraram 13\% de re-rotura nas lesões médias e 44\% nas grandes e extensas, utilizando a RNM como análise estrutural pós-cirúrgica.

Verificamos 35\% de laudos ultrassonográficos de re-rotura em nossa casuística.

Existem na literatura trabalhos que mostram não ocorrer relação entre a função e integridade da reparação do manguito rotador no pós-operatório ${ }^{(3,24)}$. Harryman et $a l^{(8)}$ relataram melhora da força, função e das atividades com o membro superior acima do nível da cabeça nos pacientes que apresentavam integridade estrutural dos tendões reparados. Também descreveram que em $87 \%$ dos pacientes que apresentavam rerotura através de imagem ultrassonográfica, não foi possível observar qualquer alteração no exame clínico, afirmando ser o US impreciso para a avaliação isolada de re-rotura. DeFranco et $a l^{(3)}$ afirmam que a melhora funcional foi mais significativa quando a integridade anatômica era totalmente restaurada, mas que lesões parciais ou re-roturas não repercutiam, necessariamente, em resultados funcionais insatisfatórios. Não encontramos significância estatística na ocorrência simultânea de resultados funcionais excelentes e bons e laudos de ultrassonografia com integridade estrutural ( $p=0,294)$. A mesma não foi encontrada nas ocorrências incoerentes de resultados funcionais excelentes/ bons com laudo de ultrassonografia demonstrando rerotura $(\mathrm{p}=0,294)$.

DeFranco et $a l^{(3)}$ descreveram que a idade é o principal fator preditivo de integridade estrutural após reparo artroscópico. Em nosso estudo a idade não teve significância estatística com a integridade pós-cirúrgica do 
manguito rotador, avaliado pelo US ( $\mathrm{p}=0,767)$. Entretanto, a idade foi fator preditivo negativo de excelente/ bom resultado funcional ( $\mathrm{p}=0,008)$.

Discrepância significativa foi observada na relação entre força pós-operatória e idade, observando-se que os pacientes com idade inferior a 60 anos apresentavam média de força maior $(5,90 \mathrm{~kg} \pm 2,49)$ que aqueles com mais de 60 anos $(4,70 \mathrm{~kg} \pm 2,23)$, $(\mathrm{p}=0,009)$.

Observou-se que pacientes com tempo médio pósoperatório mais extenso $(4,29$ anos $\pm 2,77)$, apresentaram maior incidência de laudos ultrassonográficos de re-roturas que pacientes com tempo de seguimento mais curto $(3,13$ anos $\pm 2,48)(p=0,034)$.

\section{REFERÊNCIAS}

1. Williams GR Jr, Rockwood CA Jr, Bigliani LU, Iannotti JP, Stanwoo W. Rotator cuff tears: Why do we repair them? J Bone Joint Surg Am. 2004;86(12):2764-76.

2. Veado MAC, Gomes TPO, Pinto RZA. Análise funcional e estrutural do reparo das lesões extensas do manguito rotador. Rev Bras Ortop. 2006;41(8):294-301.

3. DeFranco MJ, Bershadsky B, Ciccone J, Yum J, Iannotti JP. Functional outcome of arthroscopic rotator cuff repairs: A correlation of anatomic and clinical results. J Shoulder Elbow Surg. 2007;16(6):759-65

4. Gazielly DF, Gleyze P, Montagnon C. Functional and anatomical results after rotator cuff repair. Clin Orthop Relat Res. 1994;(304):43-53.

5. Iannotti JP, Ciccone J, Buss DD, Visotsky JL, Mascha E, Cotman K, et al. Accuracy of office-based ultrasonography of the shoulder for the diagnosis of rotator cuff tears. J Bone Joint Surg Am. 2005;87(6):1305-11.

6. Teefey SA, Hasan A, Middleton WD, Patel M, Wright RW, Yamaguchi K. UItrasonography of the rotator cuff: A comparison of ultrasonographic and arthroscopic findings in one hundred consecutive cases. J Bone Joint Surg Am. 2000;82(4):498-504.

7. Teefey SA, Rubin DA, Middleton WD, Hildebolt CF, Leibold RA, Yamaguchi K. Detection and quantification of rotator cuff tears: comparison of ultrasonographic, magnetic resonance imaging, and arthroscopic findings in seventy-one consecutive cases. J Bone Joint Surg Am. 2004;86(4):708-16.

8. Harryman DT 2nd, Mack LA, Wang KY, Jackins SE, Richardson ML, Matsen FA 3rd. Repairs of the rotator cuff: correlation of functional results with integrity of the cuff. J Bone Joint Surg Am. 1991;73(7):982-9.

9. Brandt TD, Cardone BW, Grant TH, Post M, Weiss CA. Rotator cuff sonography: a reassessment. Radiology. 1989;173(2):323-7.

10. Boileau P, Brassart N, Watkinson DJ, Carles M, Hatzidakis AM, Krishnan SG. Arthroscopic repair of full-thickness tears of the supraspinatus: does the tendon really heal? J Bone Joint Surg Am. 2005;87(6):1229-40.

11. Godinho GG. Reparação artroscópica do manguito rotador do ombro: avaliação funcional dos resultados de 87 pacientes [tese]. São Paulo: Universidade Federal de São Paulo. Escola Paulista de Medicina; 2002.

\section{CONCLUSÕES}

1 - Não há correlação estatisticamente válida entre o resultado funcional e a imagem de ultrassom $(\mathrm{p}=0,294)$.

2 - O resultado clínico nos reparos das roturas completas do manguito rotador por via artroscópica apresenta alto percentual de recuperação funcional (Constant 83,96) quando comparado com o lado contralateral.

3 - A avaliação por imagens de ultrassom apresenta altos índices de re-rotura (35\%).

4 - A força pós-operatória é maior em pacientes abaixo dos 60 anos $(p=0,002)$ e em lesões menores ou iguais a $3 \mathrm{~cm}(\mathrm{p}=0,003)$.
12. Constant CR, Murley AHG. A clinical method of functional assessment of the shoulder. Clin Orthop Relat Res. 1987;(214):160-4.

13. DeOrio JK, Cofield RH. Results of a second attempt at surgical repair of a failed initial rotator-cuff repair. J Bone Joint Surg Am. 1984;66(4):563-567.

14. Fischer BW, Gross MR, McCarthy JA, Arroyo JS. Incidence of acromioclavicular joint complications after athroscopic subacromial descompression. Arthroscopy. 1999;15(3):241-8.

15. Boehm D. Valuation of the Constant score. In: Habermeyer $P$, Magosch $P$, Lichtenberg S. Classifications and scores of the shoulder. Heidelberg: Springer; 2006. p. 204

16. Furtschegger A, Resch $\mathrm{H}$. Value of ultrasonography in preoperative diagnosis of rotator cuff tears and postoperative follow-up. Europ J Radiology. 1988;8(2):69-75.

17. Galatz LM, Ball CM, Teefey SA, Middleton WD, Yamaguchi K. The outcome and repair integrity of completely arthroscopically repaired large and massive rotator cuff tears. J Bone Joint Surg Am. 2004;86(2):219-24.

18. Prickett WD, Teefey SA, Galatz LM, Calfee RP, Middleton WD, Yamaguchi K. Accuracy of ultrasound imaging of the rotator cuff in shoulders that are painful postoperatively. J Bone Joint Surg Am. 2003;85(6):1084-9.

19. Crass JR, Craig EV, Feinberg SB. Ultrasonography of rotator cuff tears: a review of 500 diagnostic studies. J Clin Ultrasound. 1988;16(5):313-27.

20. Mack LA, Nyberg DA, Matsen FR 3rd, Kilcoyne RF, Harvey D. Sonography of the postoperative shoulder. Am J Roentgenol. 1988;150(5):1089-93.

21. Crass JR, Craig EV, Feinberg SB. Sonography of the postoperative rotator cuff. Am J Roentgenol. 1986;146(3):561-4.

22. Sugaya H, Maeda K, Matsuki K, Moriishi J, Functional and structural otcome after arthroscopic full-thickness rotator cuff repair: single-row versus dual-row fixation. Arthroscopy. 2005;21(11):1307-16.

23. Gartsman GM, Khan M, Hammerman SM. Arthroscopic repair of full-thickness tears of the rotator cuff. J Bone Joint Surg Am. 1998;80(6):832-40.

24. Anderson K, Boothby M, Aschenbrener D, Holsbeeck MV. Outcome and structural integrity after arthroscopic rotator cuff repair using 2 rows of fixation: minimum 2-year follow-up. Am J Sports Med. 2006;34(12):1899-905. 